\title{
Kapitel 16 \\ Narrative Substrukturen in den Paulusbriefen
}

\section{Vorbemerkung}

Im Laufe der in dieser Arbeit vorgelegten Analyse paulinischer Texte wurde immer wieder auf Phänomene verwiesen, die sich sehr gut als Belege für narrative Substrukturen im Sinne von Hays verstehen lassen. Daher soll im Folgenden lediglich schlaglichtartig auf einige ausgewählte Beispiele eingegangen werden, welche einzelne Möglichkeiten, die sich für die Exegese ergeben, illustrieren (Abschnitt 2 und 3). Die Betrachtung wird durch einige grundsätzliche Überlegungen zum praktischen Vorgehen (Abschnitt 4) abgeschlossen.

Bei den hier angefügten Testfällen handelt es sich um Textabschnitte, in welchen der Plot der narrativen Substruktur sich in der Regel aus der Interpretation einer expliziten Erzählung im unmittelbaren Kontext ergibt. Auch in diesen Fällen ist natürlich immer noch zu klären, $o b$ eine Erzählung im unmittelbaren Umfeld des Diskurses tatsächlich unter der Textoberfläche eine weitergehende Wirkung entfaltet, doch ist zumindest der Inhalt und Aufbau der eventuell auf diese Weise wirkenden Erzählung bekannt.

Ist dies nicht der Fall, ist die Begründung einer narrativen Substruktur weitaus schwieriger, da der Exeget zunächst die grundsätzliche Annahme zu plausibilisieren hat, dass Paulus das entsprechende Protonarrativ überhaupt in dieser Form vertrat.

Als Beispiel mag das Zitat aus Hab 2,4 in Gal 3,11 dienen: Für Hays ist es wichtig, dass Paulus hier mit ò díkaı auf den Messias Bezug nimmt. Auf diese Weise kann er argumentieren, dass das den Galatern durch die Verkündigung bekannte Motiv der Treue des Messias im Text kurz angesprochen wird (,aus

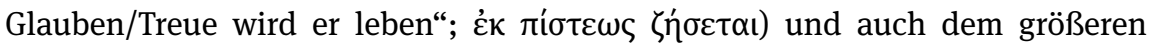
Abschnitt Gal 3,1-4,11 als Substruktur zugrunde liegt. ${ }^{1}$

Dies setzt natürlich eine sehr spezifische Lektüre dieses prophetischen Textes auf Seiten des Apostels voraus. Diese kann nun aber nicht wiederum daraus rekonstruiert werden, dass Paulus das Zitat in Gal 3,11 innerhalb eines Textes verwendet, in dem es in der Substruktur um die Treue des Messias geht, denn dies wäre ein Zirkelschluss.

Dass Paulus angesichts von Hab 2,4 das Protonarrativ des ans Kreuz gehenden Messias vor Augen hatte, bedarf also einer unabhängigen Begründung. Dies

1 Vgl. Hays, Faith, 132-141. 
zeigt unter anderem bereits die Tatsache, dass der Übersetzer des Buches in der LXX selbst offenbar keine messianische Aussageabsicht verfolgte. ${ }^{2}$

Am Beispiel von 1. Kor 8,11 und Gal 3,29 soll im Folgenden zumindest in groben Zügen angerissen werden, wie mit einiger Zurückhaltung auch dann narrative Substrukturen identifiziert werden können, wenn die „Vorlage“ nicht im unmittelbaren Kontext explizit erzählt vorliegt. Es ist jedoch zu beachten, dass in beiden Fällen das jeweils zugrunde gelegte Protonarrativ durch sehr ausführliche und deutliche Erzählfragmente aus dem vorausgehenden Text rekonstruiert werden kann.

Damit stellt sich das Verfahren als weniger problematisch dar, als wenn die „Vorlage“ aus weiter verstreuten Ereignisbezügen zusammengeflickt wird - wenn also etwa für die Erzählung von der „Treue Jesu“ vor allem auf Phil 2 verwiesen wird und davon ausgegangen wird, dass diese Erzählung durch Gal 3,1 und das

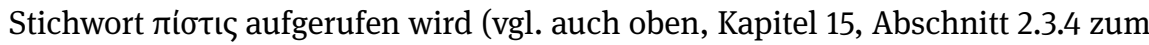
von Hays postulierten Inhalt der „gospel story,“ der jedoch nicht ausführlich aus anderen Texten abgeleitet wird).

\section{Narrative Substrukturen von nicht-narrativen Vertextungen}

Oben (Kapitel 9, Abschnitt 5) wurde eine ausführliche Analyse der expliziten Erzählung in Gal 4,3-6 geboten und aufgezeigt, wie der dadurch abgesteckte Handlungsrahmen auch im Rest des Kapitels immer wieder aufgegriffen und entfaltet wird, wobei Paulus die Vertextungsstrategie der Narration zwischenzeitlich verlässt, sich aber innerhalb dieses temporalen und sinnhaft gedeuteten Gefüges bewegt. Auch die Äußerung der Befürchtung (V. 11) auf der einen Seite und der Aufforderung (V. 12) auf der anderen machen hiervon Gebrauch.

Man könnte daher durchaus davon sprechen, dass Paulus im Hinblick auf die galatischen Gemeinden offenbar einen Ablauf an Ereignissen vor Augen hatte, von dem er sich wünschte, ihn am „Tag des Herrn“ rückblickend erzählen zu

2 Siehe dazu neuerdings die sehr umsichtige Analyse von Mulroney, Translation Style, 156-179. Hays, Faith, 135 lässt offen, ob das messianische Verständnis auf den Übersetzer selbst zurückgeht: „But whether the LXX translators intended it or not, they produced a text that is readily susceptible to messianic interpretation." Allerdings steht zu dieser Aussage der Kommentar in einer gewissen Spannung, ,the LXX rendering of Hab 2:2-3 would have appeared to Paul as unmistakably messianic. "Die spezifische, auch messianische hermeneutische Linse des Paulus ist sicherlich für sein Verständnis des Textes zu berücksichtigen (vgl. auch Hays, Faith, 136-137, der eine Parallele zum messianischen Verständnis von Gen 17,8 in Gal 3,16 zieht), zugleich scheint es jedoch sehr weit hergeholt, die Kommunikationsintention des Autors des griechischen Textes selbst gar nicht als Verständnis-Option zu berücksichtigen. 
können (vgl. auch 1. Kor 1,8, Kol 1,22; 1. Thess 5,23 für eine solche vorausschauende Perspektive; siehe aber auch 2. Kor 11,2 für Paulus als Agens).

Diese - zum Zeitpunkt des Briefschreibens noch potenzielle - Erzählung bildet die narrative Substruktur, aus welcher heraus sich auch die in V. 11 und 12 an die Oberfläche tretenden „uneigentlichen“ (d. h. nicht als tatsächlich geschehen ausgesagten) Ereignisse erklären lassen - als Bezüge auf die noch ausstehende und jetzt unsicher gewordene Handlung der Protoerzählung (V. 12) beziehungsweise genauer noch als Bezüge auf einen alternativen Handlungsstrang (V. 11), durch dessen Bewahrheitung sich auch die bisherige, explizite Erzählung (V. 3-6) als „unzuverlässig“ erweisen würde.

Am Beispiel Gal 4,11-12 zeigt sich also, wie eine Protoerzählung als narrative Substruktur, als integrierendes Prinzip für im Text erwähnte Ereignisse dienen kann, die nicht sämtliche Bedingungen erfüllen, um selbst eine Erzählung zu konstituieren.

Auch das Beispiel Röm 13,11-14 (Kapitel 14, Abschnitt 4.2.3) scheint in diese Kategorie zu fallen: Die Minimalerzählung in V. 11 kreiert ein Setting, das dann auch in den Aufforderungen in V.12-14 vorausgesetzt ist und den metaphorischen Aufforderungen Zusammenhalt verleiht.

Narrative Substrukturen können jedoch auch in Texten ausgemacht werden, in denen „Ereignisse“ überhaupt nicht im Fokus stehen. Dies wurde oben (Kapitel 8, Abschnitt 6) am Beispiel von Gal 3,28a illustriert, wo einerseits eindeutig eine deskriptive Vertextung vorliegt und so der Zustand ,in Christus“ beschrieben wird. Angesichts der begründenden Proposition V. 28b und im Kontext von Gal 3,26-29 lässt sich V. 28a jedoch konkreter als eine Betrachtung eines Endzustandes des zuvor in V. 26-27 erzählten/implizierten ${ }^{3}$ Narrativs verstehen. Es handelt sich hierbei also nicht einfach um eine Charakterisierung der „sozialen Verhältnisse“4 in christlichen Gemeinden - sondern um eine Auswertung des metaphorisch verstandenen „Anziehens“ Christi in der Taufe.

Narrative Substrukturen sind nicht auf die Herleitung von Zuständen beschränkt, die dann im Text deskriptiv durch additive Konnektoren ${ }^{5}$ entfaltet werden. Teilweise können auch im weiteren Sinn kausale Konnexionen als

3 Auch V. 26 kann als Ausdruck eines Protonarrativs (Glaube und Ko-Adoption) verstanden werden. Wirklich erzählt wird aber wohl nur in V. 27, um zu erklären, wie es zu dem in V. 26 beschriebenen Zustand kommt. Auffällig ist zumindest die Formulierung mit Aktionsnomen in V. 26 ( $\delta ı \grave{\alpha}$

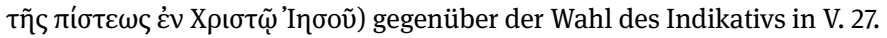

4 Noch weniger freilich ist es angebracht, die deskriptive Komponente als Ganze zu tilgen, um einen Bezug zur sozialen Struktur frühchristlicher Gemeinden zu vermeiden. So etwa Schreiner, Galatians, 259, der hierfür Gal 3,28 eine im Kontext schlicht nicht gegebene Funktion zuweist (S. 257): „Paul argues that one's ethnic background, social class, and gender are irrelevant in determining whether one is a child of Abraham.“

5 Vgl. auch GGNT 304b. Im Fall von Gal 3,28a handelt es sich um asyndetische Verknüpfung. 
Ausdruck narrativer Substrukturen verstanden werden. So bietet sich insbesondere die modal-instrumentale Konnexion an, um das in einer Erzählung temporal geordnet zum Ausdruck kommende Geschehen ohne Zeitfokus zu reformulieren. Es wird dann nicht wie in Gal 3,28a der Endzustand einer Entwicklung beschrieben oder wie in Röm 13,12-14 die Situation einer Figur zu einem bestimmten Zeitpunkt der Erzählung übernommen, sondern die Handlung als Ganzes zusammengefasst. Der Vers 1. Kor 8,11 (vgl. Kapitel 5, Abschnitt 4.2) demonstriert dies mustergültig. Hier geht eine konkrete Ereignisfolge voraus. Diese wird zwar auch nicht explizit erzählt, da sie lediglich im Rahmen eines Konditionalgefüges erwogen wird, der Ereigniszusammenhang ist jedoch ganz klar als eine potenzielle Erzählung erkennbar (vgl. oben zu Gal 4,11-12). In V. 11 wird dann der temporale Fokus durch die modal-instrumentale Konnexion aufgelöst.

Im konkreten Textbeispiel ist sowohl die stärker temporal fokussierte „Superstruktur“ - so Hays’ despektierliche Bezeichnung für explizit(er)e Erzählstrukturen (Kapitel 2, Abschnitt 4) - als auch die für V. 11 übernommene narrative Substruktur für das Erreichen der Kommunikationsintention von Bedeutung: Während (a) die temporale Ordnung im vorausgehenden Text einen generellen Ablauf skizziert, welcher dem Leser erlaubt, sich in dieser Situation wiederzuerkennen, spielt dann in V. 11 die Sequenz keine Rolle mehr. Vielmehr wird nun (b) der Plot der potenziellen Erzählung ausgewertet und die Relation der einzelnen Beteiligten durch eine Mittel-ERGEBNIS-Konnexion zum Ausdruck gebracht. Das so herausdestillierte Verhältnis wird wohl den „Starken“ in der Alltagserfahrung und auch in der weniger auf diesen Aspekt kondensierten vorausgehenden angedeuteten Ereignisfolge so nicht klar vor Augen gestanden haben. Temporale und nicht-temporale Fokussierung greifen hier also ineinander, um die Leser zu einer Neubewertung ihrer Situation und so zu einem veränderten Handeln zu bewegen. Der Blick auf die Textfunktion zeigt also wieder, dass implizite und explizit(er)e Erzählungen - narrative Super- und Substrukturen - nicht gegeneinander ausgespielt werden dürfen.

Ganz ähnlich kann die Handlung einer Erzählung auch dahingehend ausgewertet werden, dass sie als narrative Substruktur ein konditionales Verhältnis in einer argumentativen Vertextung stützen. Besonders klar ersichtlich ist dies in Gal 3,29. Einerseits ist dort (a) die Protasis „Wenn ihr aber des Christus seid ... “

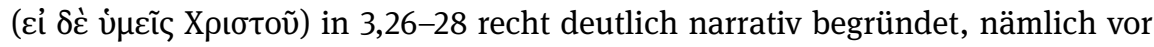
allem durch den Rückverweis auf die Taufe in V. 27 und die Entfaltung der damit verbundenen Metapher des „Anziehens.“ Aber auch (b) der Zusammenhang des Wenn-Dann-Verhältnisses selbst - warum also aus der Protasis die Apodosis zu folgen hat - ist letztlich durch ein vorausgesetztes Protonarrativ gestützt, welches in Kapitel 3 des Galaterbriefes fragmentarisch zum Ausdruck kommt.

Hier liegt also (vgl. Kapitel 15, Abschnitt 5.2) die narrative Substruktur nicht zusätzlich als explizite Erzählung im Kontext vor. Aufgrund der deutlichen 
Stichwortverbindungen innerhalb eines so kurzen, zusammengehörigen Abschnitts, lässt sich das vorausgesetzte Narrativ dennoch sehr gut rekonstruie-

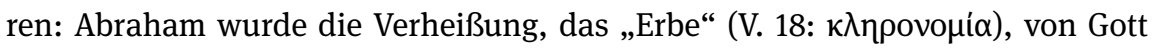
geschenkt. Diese Verheißung gilt dem wahren „Nachkommen“ (und Erben), welcher Christus ist (V. 16). Das Auftreten des Gesetzes (V. 17-25) hat durchaus eine Funktion, die nun jedoch beendet ist, da Christus, der Nachkomme und Erbe, offensichtlich „gekommen“ ist (vgl. die elliptische Erzählung in 3,23-25; siehe Kapitel 8, Abschnitt 2.2). Nach dessen „Kommen“ und Kreuzigung, wurde dies den Galatern von Paulus vermittelt (V. 1), es kam zu einer gläubigen Reak-

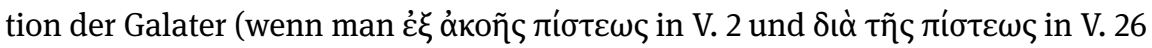
so verstehen darf) und zu deren Taufe (V. 27). Durch diesen Vorgang wurden sie derart mit Christus verbunden (V. 27-28), dass sie - so setzt die Protasis in V. 26 ganz selbstverständlich voraus - wie er „Söhne Gottes“ sind.

Es ist vor dem Hintergrund dieses Protonarrativs, dass aus der Zugehörigkeit zu

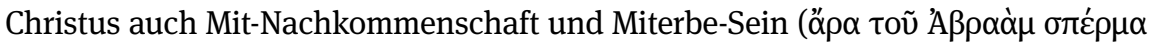

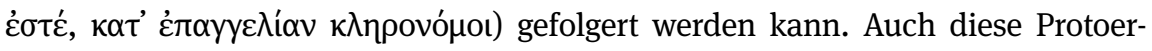
zählung ist lediglich aus Fragmenten rekonstruiert. Anders als im Fall eines die Treue Jesu betreffenden Narrativs ist diese Rekonstruktion jedoch nicht auf Textabschnitte anderer Schreiben oder hypothetischer mündlicher Vorstufen angewiesen.

\section{Narrative Substrukturen von narrativen Vertextungen: Beispiel Gal 4,1-7}

Vor dem Hintergrund von Gal 3,26-29 bietet es sich an, direkt zu 4,1-7 überzugehen (zu Gal 4 insgesamt vgl. Kapitel 9, Abschnitt 5). Auf den ersten Blick scheint es, als würde Gal 4,7 ganz analog zu Gal 3,29 argumentativ vertextet eine Zusammenfassung und Explikation des vorausgehenden Narrativs vornehmen. Zusätzlich bietet es sich an, Gal 4,3-6 als eine Erzählung zu verstehen, deren Aufbau dem iterativ erzählten Narrativ in 4,1-2 folgt, also dessen Plot als narrative Substruktur aufgreift. Andere Exegeten sehen in der nach antikem Erbrecht ungewöhnlichen Rede von einem festgesetzten Zeitpunkt in V. 2 eine Angleichung an die Erzählung in Gal 4,3-6, sodass man eher umgekehrt davon sprechen müsste, dass diese Erzählung eines konkreten Ereigniszusammenhangs die Substruktur für die Erzählung des allgemeingültigen Zusammenhangs der Ereignisbündel in 4,1-2 lieferte. ${ }^{6}$ Wieder andere nehmen als gemeinsamen Hintergrund eine weitere

6 Vgl. etwa Moo, Galatians, 259, der von einem „backreading of the application into the illustration" spricht. 
narrative Substruktur an, nämlich die Exodus-Erzählung, in welcher Befreiung und Adoption zusammenfallen. ${ }^{7}$

Ganz grundsätzlich muss zunächst festgehalten werden: Die Option, dass eine Erzählung eine andere Erzählung als narrative Substruktur aufweist, ist in der Tat nicht von der Hand zu weisen. In Gal 3,6 wird sogar auf eine einen Ereigniszusammenhang betreffende, lediglich implizierte Antwort der Galater auf die Frage in 3,5 Bezug genommen. Die Handlung der von Paulus als Antwort vorausgesetzten Erzählung wird dann von ihm mit einem anderen Narrativ (aus Gen 15,6 zitiert) korreliert. Es liegt also durchaus nahe, Gal 4,1-2 als die narrative Substruktur für die darauffolgende narrative Vertextung zu verstehen.

Die Passage Gal 4,1-7 weist bei näherer Betrachtung jedoch auch gerade darauf hin, dass man mit der Annahme narrativer Substrukturen hinter Erzählungen bei Paulus selbst dann äußerst vorsichtig sein muss, wenn die angebliche Vorlage im Kontext explizit erzählt wird. Es soll im Folgenden gezeigt werden, dass das weit verbreitete Verständnis von Gal 4,3-6 als eine der generellen Handlungsstruktur von 4,1-2 folgende Erzählung so nicht haltbar ist. Auch wenn die Kategorie der narrativen Substruktur hier durchaus eine gewisse Berechtigung hat, so muss sie doch sehr differenziert in Anschlag gebracht werden. Es kann auf jeden Fall nicht die Rede davon sein, dass V. 3-6 schlicht dem Plot von V. 1-2 folgt.

Ein erster Hinweis auf die Problematik eines solchen Verständnisses liefert Gal 4,7. Zunächst fasst V. 7a die Handlung der Verse 3-6 mit Fokus auf den End-

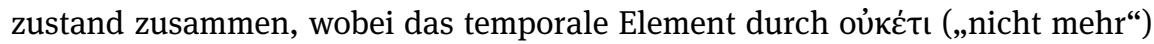

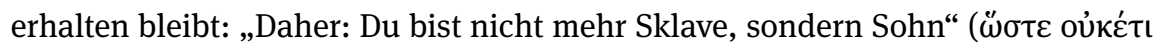

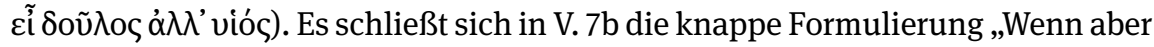

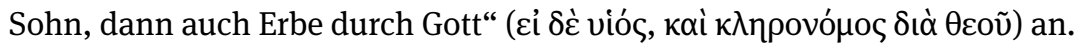

Die Rede vom $\kappa \lambda$ поvó $\mu$ oৎ verweist nun zurück auf V. 1, und genau dadurch entsteht eine gewisse Spannung: In V. 1-2 liegt nämlich eine Handlung vor, in welcher ein Sohn zur „Mündigkeit“ gelangt und somit den ehemaligen Status

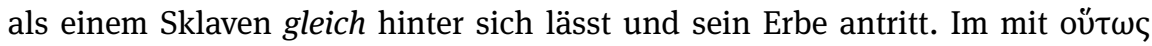
eingeleiteten Übergang zu „uns“ in V. 3 werden die Elemente von Unmündigkeit und Versklavung nun noch deutlich stärker verknüpft (Unmündigkeit ist nun Versklavung) und der Übergang vom vormaligen in den späteren Zustand ist nun nicht mehr der festgelegte Zeitpunkt des tatsächlichen Antritts des Erbes (V. 2), sondern der Zeitpunkt der Adoption zu Söhnen (V. 5-6).

Das Konditionalgefüge in V. 7b eignet sich daher auf den ersten Blick weder gut dazu, die Handlung von 4,1-2 noch die von 4,3-6 zusammenzufassen. In keinem der beiden Fälle ist sowohl Sohnschaft vorausgesetzt, Erbenschaft aber daraus abgeleitet.

7 Scott, Adoption. Vgl. auch Hafemann, „Exile.“ 
Denn der Sohn in 4,1-2 ist natürlich auch schon vor dem festgesetzten Zeitpunkt in

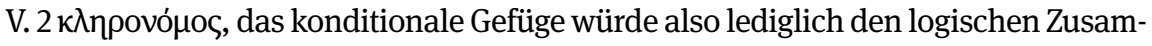
menhang des Ausgangsstadiums des Geschehens von V. 1-2 ausdrücken. Aus dieser Erzählung folgt daher nicht, dass ein Sohn ein Erbe ist und ihre Handlung lässt sich daher kaum durch den Wenn-Dann-Zusammenhang in 7b zusammenfassen.

Und die Erzählung 4,3-6 läuft ja gerade auf das Verlassen der Unmündigkeit hinaus, welche aufgrund der Gleichsetzung von Versklavung und Unmündigkeit in V. 3 als ein Verlassen des unfreien Standes charakterisiert wird und als Adoption durch Gott. Die Erzählung mündet daher offenbar im Endzustand der Sohnschaft. Auch aus dieser Erzählung folgt daher nicht, dass ein Sohn auch ein Erbe ist.

Möchte man den Ereigniszusammenhang des Konditionalgefüges mit der Erzählung in den Versen 3-6 zusammenbringen, so könnte man, isoliert betrachtet, höchstens erwägen, ob V. 7b als eine den Endzustand von V. 3-6 weiterführende Implikation zu betrachten sei: „Ihr seid nicht nur (wie die Erzählung zeigt) Söhne, sondern als Söhne (automatisch) auch Erben!“ Die Funktion von 4,7b wäre dann ganz ähnlich wie die von 3,29, wo ja auch das zuvor Festgehaltene weitergeführt wird. Genau diese Parallele macht eine solche Interpretation aber auch sehr unwahrscheinlich: Denn gerade weil in 3,29 das Erbe-Sein bereits gefolgert worden war, würde die Charakterisierung als „bloßer“ Erbe in 7b kaum einen argumentativen Fortschritt darstellen.

Dass die Galater auch Erben sind, braucht daher eigentlich nicht extra geschlussfolgert zu werden. Dass die Konditionalperiode diesen Zusammenhang aufgreift, hat daher also wohl den Sinn, die Parallelität zwischen den Erzählfiguren in V. 1-2 und 3-6 aufzuzeigen: In beiden Fällen geht es um Söhne - und daher auch um Erben. Wenn die Galater Söhne sind, so ist ihnen automatisch auch ein Erbe versprochen.

Daraus folgt, dass auch für sie der in V. 1-2 dargestellte temporale Zusammenhang gilt. Sie werden somit dazu aufgefordert, zu reflektieren, wann sie ihr Erbe auch antreten. Die Implikation der Gegenüberstellung von V. 1-2 mit 3-6 ist also, dass zu einem Abgleich dessen aufgefordert wird, was in beiden Fällen im Moment des Umschlagens von einem „Früher“ zu einem „Nun“ geschieht.

Dass die galatischen Christen sich (im „Jetzt“!) in der Situation der Unmündigkeit eines Sohnes befinden, kann wohl kaum die Aussageintention sein, vor allem auch da V. 4 das Element des temporalen Umschlagpunktes so explizit auf-

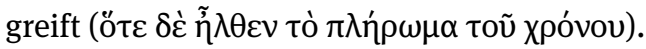

Da ihre Adoption als Söhne in der Erzählung V. 3-6 zugleich als Übergang von der Unmündigkeit zur Mündigkeit und von der Sklaverei in die Freiheit charakterisiert wird - also ganz analog zu einem Sohn, der sein Erbe auch antritt liegt die Schlussfolgerung auf der Hand: mit der Adoption ist den Galatern auch bereits das Erbe anvertraut worden. 
Die Spannung, die zwischen 4,1-2 und 4,3-6 besteht, ergibt sich folglich nicht daraus, wie manche meinen, dass Paulus das Handlungsmuster aus 4,1-2 nicht konsequent „anwenden“ würde, die Verse 1-2 also eine narrative Substruktur für die Erzählung 3-6 lieferten, Paulus aber dadurch den Diskurs nicht strukturieren, sondern eher zersetzen würde.

Vielmehr erzählt Paulus in Gal 4,3-6 eine den Galatern bereits bekannte Geschichte von ihrer Bekehrung auf eine ganz spezifische Weise, wobei das erstaunliche Moment nicht darin besteht, dass dieses Geschehen als Adoption geschildert wird (die ist in 3,26 ganz selbstverständlich vorausgesetzt), sondern darin, dass dieser Vorgang zugleich Analogien aufweist (neu gewonnene „Freiheit“) zu einem iterativen Erzählen vom Übergang eines unmündigen Erben zu einem mündigen, das Erbe antretenden Erben.

Damit klärt sich auch das Verhältnis von Gal 4,1-7 zu Gal 3,29. Paulus liefert nicht einfach aufgrund des Stichwortes $\kappa \lambda$ пovó $\mu$ oৎ eine weitere Illustration aus demselben semantischen Feld. Eher noch könnte man vermuten, dass er den Aspekt der Gottessohnschaft aus 3,26 - der dort recht unvermittelt erscheint, allerdings wichtig für den Zusammenhang des konditionalen Gefüges in 3,29 ist nun ausführlicher begründen möchte. Dazu passt aber weder die Zwischenschaltung von 4,1-2 noch die Art und Weise der Narration von 4,3-6, wo der eigentliche Akt der Adoption nämlich bloß elliptisch erzählt wird. Vielmehr wendet sich Paulus in Gal 4,1-7 einem Aspekt zu, der in Gal 3,29 noch gar nicht zur Sprache kam: Dem Zeitpunkt des Antretens des Erbes.

Gal 4,1-2 liefert also in gewisser Hinsicht durchaus eine „narrative Substruktur“ für 4,3-6: die Art und Weise, wie V. 3-6 erzählt wird, orientiert sich ganz offensichtlich an der Ausgestaltung von V. 1-2. Es ist jedoch zu beachten, dass 4,1-2 nicht einfach die Handlung von 4,3-6 vorgibt, denn die Erzählungen haben dennoch sehr unterschiedliche Themen: Gal 4,1-2 ist eine Erzählung über einen Sohn, der sein Erbe antritt, Gal 4,3-6 ist eine Erzählung über Sklaven, die zu Söhnen adoptiert werden. Die Übertragung von Elementen aus der ErbschaftsErzählung auf die Adoptions-Erzählung macht deutlich, dass auch in der letztgenannten das Antreten des Erbes bereits enthalten ist. Als narrative Substruktur tritt V. 1-2 also insofern auf, als das Erzählen selbst beeinflusst wird. Die „Unstimmigkeiten“ sind kein erzählerisches Missgeschick, sondern genau diejenigen Elemente, in denen die Substruktur überhaupt erkennbar wird. Es ist gerade diese Verfremdung der gängigen Adoptions-Erzählung, die zur Reflexion über das Antreten des Erbes anregt und somit die Kommunikationsintention verwirklicht.

Erkennbar wird diese Absicht dann auch im den vorangehenden Text kommentierenden Vers 7. Während Gal 4,7a die Erzählung in V. 3-6 im Hinblick auf den unstrittigen Plot der Adoption zusammenfasst (,nicht mehr Sklave, sondern Sohn“), verweist V. 7b auf die Ähnlichkeit zu einer anderen Erzählung in V. 1-2, 
nämlich was die Erzählfiguren angeht („Söhne und somit auch designierte Erben“). Diese Erzählung hat jedoch einen anderen Gegenstand, sodass zu einer Übernahme von Elementen aus diesem Plot aufgefordert wird, die Galater im „Jetzt“ also charakterisiert werden als (nicht nur Söhne, nicht nur designierte Erben, sondern als) das Erbe angetreten habende, mündige Erben. (Vgl. auch oben, Kapitel 8, Abschnitt 4.4.2 zur Textfunktion von Gal 3,23-25).

\section{Das Erklärungspotenzial der Annahme einer narrativen Substruktur}

\subsection{Grundsätzliches}

In wissenschaftstheoretischen Kategorien ${ }^{8}$ gesprochen weist die These einer narrativen Substruktur für einen bestimmten paulinischen Text eine solide „PriorWahrscheinlichkeit“ auf, insbesondere wenn es sich hierbei um Passagen handelt, die im unmittelbaren Kontext narrativer Vertextungen anzusiedeln sind. Es ist dann, mit anderen Worten, durchaus eine naheliegende Vermutung, dass die Erzählung unter der Textoberfläche auch noch im weiteren literarischen Kontext ihre Kreise zieht.

Dass hiermit tatsächlich grundsätzlich gerechnet werden muss, ergibt sich einerseits aus den theoretischen Überlegungen zum Zusammenhang von expliziter Erzählung und mentaler Narrativisierung und Simulation (Kapitel 9; vgl. auch in Kapitel 15, Abschnitt 5.2) als auch aus den zum Teil sehr deutlich aus dem Text zu erschließenden impliziten Erzählungen (vgl. Kapitel 10 und 11 für Tiefenbohrungen und Kapitel 12 bis 14 für eine schlaglichtartige Erhellung der Bandbreite). ${ }^{9}$

8 Für eine Explikation des in diesem Abschnitt Gesagten siehe vor allem Heilig und Heilig, „Historical Methodology. “ Ein erster Versuch, die Kritierien von Hays, Echoes vor diesem bayesianischen Hintergrund zu analysieren, bietet Heilig, „Methodological Considerations.“ Heilig, Paul's Triumph überträgt die theoretischen Überlegungen auf die Frage nach der Semantik von 2. Kor 2,14. Dabei wird gänzlich auf mathematische Ausdrucksweise verzichtet, aber die Grundstruktur des Buches an Bayes' Theorem ausgerichtet (vgl. Schmeller, „Rezension“ für eine sehr positive Bewertung des Vorgehens). Siehe auch Heilig, „Argumentation“ - eine kritische Analyse des „Intelligent Design“-Arguments - für die Bewertung einer historischen Rekonstruktion außerhalb des Bereichs der neutestamentlichen Wissenschaft.

9 Mit anderen Worten: Das Erklärungspotenzial der Hypothese impliziter Narrative ist zumindest bei manchen dieser Texte so hoch, dass selbst eine sehr zurückhaltende ursprüngliche Einschätzung der Hintergrundwahrscheinlichkeit dieser These im Ergebnis die Schlussfolgerung zulässt, dass tatsächlich an einigen Stellen in den Paulusbriefen narrative Substrukturen auszumachen sind. Diese, aufgrund eindeutigerer Belege getroffenen, Konklusionen heben wiede- 
Damit im weiteren Verlauf der Exegese auch weniger klare Fälle mit guten Gründen als Manifestationen narrativer Substrukturen identifiziert werden können, muss noch ein weiterer Aspekt erfüllt sein, ein Erklärungspotenzial, welches die Hypothese gegenüber alternativen Erklärungen überlegen erscheinen lässt. Es genügt folglich nicht, wenn die Annahme einer narrativen Substruktur einzelne Formulierungen zufriedenstellend erklären kann. Vielmehr darf und muss an die Annahme einer narrativen Substruktur für eine konkrete Passage der Anspruch gestellt werden, dass sie die tatsächlich vorliegende Ausgestaltung der Textoberfläche als Ganzes und besser erklärt als andere Hypothesen.

Diese Formulierung setzt voraus, dass vor Betrachtung dieses Erklärungspotenzials beide Hypothesen - die Annahme einer narrativen Substruktur ebenso wie deren Ablehnung - gleich plausibel sind. Geht man davon aus, dass narrative Substrukturen quasi die Standard-Option für Passagen in den Paulusbriefen darstellen, kann man im Einzelfall natürlich auch ein solches Phänomen schlussfolgern, wenn das Erklärungspotenzial geringer ist als im Fall der Alternativannahme. Umgekehrt ist auch ein lediglich „,besseres“ aber nicht hervorragendes Erklärungspotenzial für Exegeten, die narrative Substrukturen für ein Ausnahmephänomen halten, kaum hinreichend für eine solche Schlussfolgerung. Hintergrundwahrscheinlichkeit und Erklärungspotenzial sind immer (gleichwertig!) im Zusammenspiel und immer im Vergleich mit alternativen Erklärungen zu bewerten.

Mit welchen Vorannahmen man an den Text herantritt, ist dabei in hohem Maß auch eine subjektive Einschätzung und hängt nicht zuletzt damit zusammen, wie viele - vielleicht eindeutiger zu beurteilende - Texte man zuvor bereits untersucht und entsprechend klassifiziert hat. Auch wenn es wenig Sinn macht, hier nach statistischer Präzision zu streben, ist es doch zumindest eine hilfreiche Stütze, sich die Frage zu stellen, ob man von narrativen Substrukturen in mehr oder weniger als der Hälfe der Fälle der zu beurteilenden Textteile ausgeht. ${ }^{10}$ Entsprechend ist dann ein geringerer oder größerer Anspruch an das Erklärungspotential zu stellen.

Der Aspekt des Erklärungswertes einer Hypothese wird über die Beantwortung der Frage bestimmt, welche konkrete Form des Textes man erwarten würde, falls die jeweilige Annahme tatsächlich zutreffend wäre - beziehungsweise wie „erwartbar“ die tatsächliche Formulierung vor diesem Hintergrund wäre. ${ }^{11}$ Es

rum die neue Hintergrund-/Prior-Wahrscheinlichkeit der These soweit, dass nun auch weniger eindeutige Belege, also Fälle, in denen das Erklärungspotenzial der zu prüfenden Hypothese geringer ist, eventuell positiv bewertet werden können. Vgl. zum wissenschaftstheoretischen Hintergrund dieses Vorgehens Heilig und Heilig, „Historical Methodology.“

10 Vgl. Heilig und Heilig, „Historical Methodology,“ 141-142.

11 Im Rahmen der bayesianischen Bestätigungstheorie spricht man dann von der sogenannten „likelihood.“ Vgl. hierzu Heilig und Heilig, „Historical Methodology.“ 
genügt folglich nicht, dass im Text einzelne Elemente identifiziert werden können, welche mit der Annahme einer zugrundeliegenden Erzählung „vereinbar“ wären. (Vgl. etwa das Element eines festgelegten Zeitpunks in Gal 4,2, welches gut zur Exodus-Erzählung „passen“ würde.)

Vielmehr muss auch begründet werden, dass der vom Autor gewählte Text eine plausible Wahl ${ }^{12}$ im Vergleich zu alternativen - möglichen, aber letztlich nicht verwirklichtem - Ausdrucksweisen darstellt. So lässt sich etwa an Wrights Interpretation von Röm 6-8 die grundsätzliche kritische Anfrage richten, weshalb die traditionsgeschichtliche Analyse dieses Abschnitts nicht mehr Exodus-Material zu Tage fördert. ${ }^{13}$ Inwiefern, so könnte man etwa fragen, weist der Text Merkmale auf, die im Rahmen einer Orientierung an der Befreiung Israels aus Ägypten eher zu erwarten wären als unter der Voraussetzung, dass die Alltagserfahrung einer Befreiung eines Sklaven den Hintergrund bildet? ${ }^{14}$

Diese Frage ist wie gesagt unabhängig davon $\mathrm{zu}$ beantworten, für wie plausibel man es grundsätzlich (d. h.: unabhängig von der tatsächlich anzutreffenden Wortwahl) hält, dass Paulus sich in seinem Schreiben an die Römer auf die Erfahrung der Knechtschaft des Volkes Gottes in Ägypten oder der Sklaverei seiner Zeit bezieht. Beide Faktoren sind vollständig unabhängig von einander zu erheben und mit dem gleichen Gewicht für die Einschätzung der konkurrierenden Thesen zu berücksichtigen. Entscheidend ist das Gesamtbild. Es kann durchaus Alternativen zur plausibelsten Hypothese geben, welche sie in einem der beiden Parameter deutlich übertreffen. Ein stichhaltiges Argument muss daher immer mit offenen Karten spielen und beide die Entscheidung beeinflussenden Einschätzungen mit Verweis auf die Evidenz spezifizieren.

12 Die Bestimmung, ob ein Ausdruckselement eine plausible Wahl für eine postulierte Bedeutung ist oder nicht, wird im Normalfall unter der Voraussetzung vorgenommen, dass ein Sprecher verstanden werden will und über die geeigneten Strategien verfügt, um sich verständlich zu machen. Vgl. GGNT 314 für die damit zusammenhängenden „Kommunikationsprinzipien.“ Beim Erzählen eines unzuverlässigen Erzählers (vgl. Kapitel 3, Abschnitt 5.4) können diese Prinzipien in verschiedener Hinsicht gestört sein.

13 Siehe die fünf Traditionsfelder, die Vollenweider, Freiheit, 360 für Röm 7-8 identifiziert. Zu Röm 6 siehe bereits oben, Kapitel 15, Abschnitt 4.5.1.

14 Vgl. etwa Wischmeyer, „Biblical Hermeneutics.“ 


\subsection{Beispiel 2. Kor 2,14}

\subsubsection{Der Übergang von V. 13 zu V. 14 aus narratologischer Sicht}

Das heuristische Potenzial auf der einen Seite und Gefahren sowie Beschränkungen des Postulats narrativer Substrukturen auf der anderen Seite sollen vor dem hier kurz angerissenen, an anderer Stelle ausführlich behandelten methodologischen Hintergrund am Beispiel von 2. Kor 2,14 erläutert werden. ${ }^{15}$

In 2. Kor 2,12 erzählt Paulus von seiner Reisebewegung nach Troas (E $\lambda \theta \dot{\omega} v$

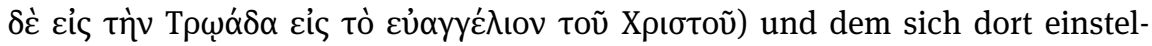

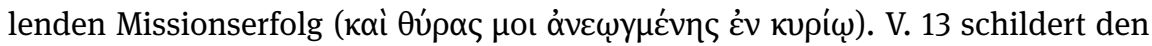
unerwarteten weiteren Verlauf: Da sich dort, anders als erwartet, Titus nicht ein-

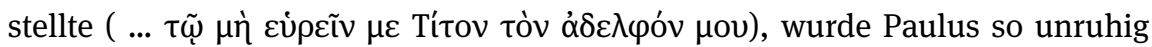

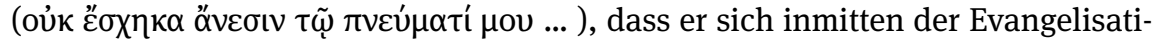

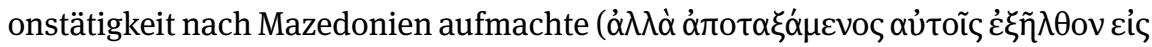

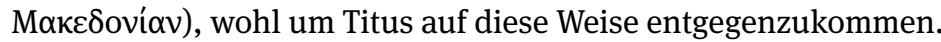

Das Resultat dieser erneuten Reisebewegung wird nicht direkt erwähnt.

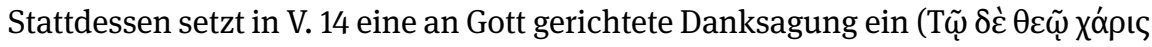
$\tau \tilde{\omega}$... ), die in eine Apologie des apostolischen Dienstes des Paulus übergeht. Erst in 2. Kor 7,5-7 (Kà̀ yà dass Paulus in Mazedonien tatsächlich auf Titus traf, dieser gute Neuigkeiten aus Korinth überbrachte und dadurch Paulus tröstete.

Der Abschnitt 2. Kor 2,14-7,4, von Bengel als nobilissima digressio bezeichnet, ${ }^{16}$ wurde später von anderen Exegeten nicht mehr als Abschweifung, sondern als Fragment eines anderen Briefes verstanden. $\mathrm{Zu}$ abrupt sei der Übergang von 2,13 zu 2,14, als dass es sich hierbei um den ursprünglichen Text handeln könnte. ${ }^{17}$ Das gewichtigste Argument, das für diese Position vorgebracht wird, stellt jedoch die „,continuity of the narrative“ der Verse 2,12-13 und 7,5 dar. ${ }^{18}$ Narratologische Überlegungen im weiteren Sinne wurden bisher in der Erforschung dieses Textes also primär dafür bemüht, die literarische Uneinheitlichkeit des Abschnittes zu begründen.

Eine sorgfältige Analyse der Erzählung 2,12-13 vor dem Hintergrund der zuvor gemachten Äußerungen weist jedoch in eine ganz andere Richtung: Schon bei der ersten Nennung von M $\alpha \kappa \varepsilon \delta o v i \alpha(1,16)$ musste sich Paulus mit dem Vorwurf der „Leichtfertigkeit“ (1,17: غ่ $\lambda \alpha \varphi \rho i ́ \alpha)$ auseinandersetzen. Auch dort ging es bereits um die Reisebewegungen des Paulus, deren Abweichen von ursprünglichen

15 Details zur Exegese der einzelnen Verse finden sich bei Heilig, Paul's Triumph.

16 Bengel, Epistolas, 244.

17 Siehe die neuerliche ausführliche Darbietung der Argumente bei Duff, Moses, 18-51.

18 Welborn, „Broken Pieces,“ 569. 
Plänen die Überlegungen des Paulus als „fleischlich“ erschienen ließ (vgl. 1,17: ク̋

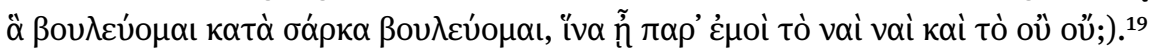
Einen analogen Einwurf kann Paulus nun auch nach 2,13 erwarten, wo ihn seine Sorge um die Korinther eine von Gott eröffnete missionarische Möglichkeit aufgeben lässt. ${ }^{20}$

Paulus reagiert auf die von ihm angenommene Reaktion auf seine Erzählung, indem er sich der Metapher des römischen Triumphzugs bedient: der in V. 14 geäußerte Dank gilt Gott, ,der uns allezeit in Christus im Triumphzug prä-

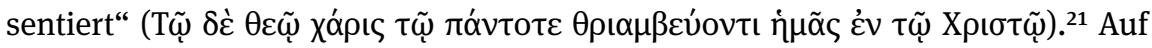
diese Weise greift Paulus einerseits die Wahrnehmung der Korinther auf: In der Metapher entsprechen sie der implizierten Volksmenge, welche dem Spektakel der vorüber geführten Kriegsgefangenen beiwohnt. ${ }^{22}$

Zugleich kommt es auf mehrfache Weise zu einer Transformation der Art und Weise, wie Paulus wahrgenommen wird: Der Triumphator, also derjenige, der die Bewegung der im Triumphzug Mitgeführten veranlasst, ist nämlich niemand anderes als Gott selbst. Wer an Paulus' Missionsbewegungen Anstoß nimmt, kritisiert damit also letztlich Gott. ${ }^{23}$

Zugleich spielt Paulus mit einer gewissen Vieldeutigkeit der Rolle des Gefangenen im römischen Triumphzug. Die Quellen zeichnen, entgegen der Annahme der meisten modernen Exegeten, ${ }^{24}$ durchaus nicht ein durchgängiges Bild von geschändeten, in Ketten gelegten, gramgebeugten, zum Tode bestimmten Gefangenen. Nicht selten wird auch die Nobilität der Präsentierten hervorgehoben und von den Zuschauern bestaunt - was den Ruhm des Triumphators nur noch steigert (es sei denn, die Gefangenen stehlen ihm die Show). ${ }^{25}$ Im Triumphzug einher geführt zu werden, hat also durchaus auch eine „positive“ Komponente ${ }^{26}$ - die

19 Zum Abschnitt und der Syntax von 2. Kor 1,17 vgl. bereits oben, Kapitel 14, Abschnitt 3.1.1.

20 Zur Begründung siehe Heilig, Paul's Triumph, Kapitel 3, Abschnitt 2.6.

21 Zur lexikalischen Bedeutung des Verbs in der Antike siehe Heilig, Paul's Triumph, Kapitel 2.

22 Für die Leserwirkung siehe Heilig, Paul's Triumph, Kapitel 7, Abschnitt 3. Vgl. auch, was Köppe und Kindt, Erzähltheorie, 124 zur Figurenanalyse (vom ,internen Standpunk“) schreiben: „Leser haben zu allen Zeiten in den Anliegen und Problemen von Figuren ihre eigenen Probleme wiedererkannt. Literarische Figurendarstellungen werden in diesem Sinne bisweilen als ein Simulations- oder Experimentierfeld angesehen, in dem allgemeinmenschliche Möglichkeiten dargestellt und ausprobiert werden.“

23 Vgl. Heilig, Paul's Triumph, 254-255.

24 Etwa Hafemann, Suffering.

25 Vgl. Heilig, Paul's Triumph, 257-259.

26 Diese Beobachtung scheint mir nach wie vor die kritische Anfrage von Schmeller, „Rezension,“ 341 zu beantworten, der in dieser Interpretation wohl keinen ausreichend positiven Grund für den paulinischen Dank zu sehen scheint: „[Heiligs Deutung] kann nicht erklären, warum 
es Paulus erlaubt, das Bild mit einem Dank zu begleiten. Expliziert wird dieser positive Aspekt durch die koordinierte Partizipialphrase „... und der den Geruch

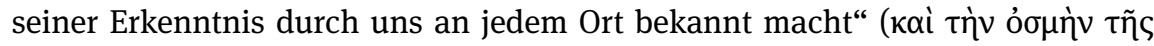

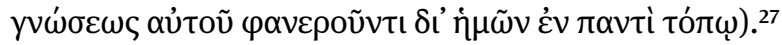

Eine sorgfältige narratologische Analyse von 2,12-13, welche diese Erzählung zudem im größeren kommunikativen Kontext der korinthischen Korrespondenz wahrnimmt, spricht also (a) vielmehr für die Einheitlichkeit von 2. Kor 1-7.28 Zugleich demonstriert die sorgfältige Wahl und Konfiguration (durch die Angaben und Ergänzungen zum Verb) ${ }^{29}$ der Metapher, mit welcher Paulus sowohl die Einstellung der Korinther aufzunehmen und zu korrigieren versucht, als auch deren Platzierung im Diskurs, (b) wie aufmerksam Paulus erzählt. ${ }^{30}$

Die Kategorie der (c) narrativen Substruktur stellt sich folglich auch hier als heuristisch wertvolles Werkzeug dar: Anstatt einen unbedachten Übergang im Diskurs oder sogar einen literarischen Bruch postulieren zu müssen, bietet die Annahme, dass Paulus auch in der Metapher vom Triumphzug die Erzählung aus 2,12-13 noch vor Augen hat, eine weitaus befriedigendere Erklärung des Textes. ${ }^{31}$

\subsubsection{Die Gefahr der narrativen Überinterpretation}

\subsubsection{Weihrauchträger in V.14b?}

2. Kor 2,14 dient zugleich jedoch auch als Mahnung dafür, dass die genaue Gestalt, Ausdehnung und Funktion einer narrativen Substruktur im Einzelfall immer wieder erneut plausibilisiert und bestimmt werden muss.

Dies gilt bereits für die zweite Partizipialkonstruktion in V. 14: Eine recht große Zahl Exegeten hat erwogen, dass Paulus hier das Bild des Triumphzugs fortsetzt.

sich Paulus mit dieser Metapher in der Rolle eines besiegten Feinds darstellen sollte, der zwar vielleicht nicht hingerichtet wurde, der aber auch beim Triumphzug ein Feind des Triumphators blieb, während in der Realität Paulus selbst eindeutig auf die Seite Gottes gewechselt war. Diese Überlegung ist eine ernste Anfrage an das ,Explanatory Potential` der von H. vertretenen Deutung.“

27 Vgl. Heilig, Paul's Triumph, 255-256.

28 Dies zeigt sich bereits daran, dass trotz der in diesem Fall ungehindert waltenden Kreativität bisher kein überzeugender Vorschlag für den ursprünglichen Anlass von 2. Kor 2,14 präsentiert werden konnte (vgl. Heilig, Paul's Triumph, 244-245).

29 Vgl. Heilig, Paul's Triumph, Kapitel 6.

30 Und wie aufmerksam er den römischen Kontext seiner Gemeinden wahrnimmt. Vgl. Heilig, Paul's Triumph, 275-277.

31 Diese Aussage setzt natürlich eine entsprechende Prüfung der Argumente voraus, die zugunsten der Alternativhypothesen vorgebracht werden. Siehe hierfür im Detail Heilig, Paul's Triumph, 146-191. 
So hat Breytenbach postuliert, dass die Metapher vom Triumphzug nicht voraussetze, dass Paulus als Gefangener an diesem teilnehme, nur dass er vormals wohl bei Damaskus - als Feind Gottes diesem unterlegen sei. ${ }^{32}$ Dies eröffnet die Möglichkeit, dass sich Paulus nun als ein mit dem siegreichen General freudig einherschreitender Weihrauch-Träger darstellen könnte, ohne dass dadurch ein Bruch im Bild anzunehmen wäre. ${ }^{33}$

Auch abgesehen davon, dass Breytenbachs Rekonstruktion der antiken Bedeutung von $\theta \rho \iota \alpha \mu \beta \varepsilon u ́ \omega$ nicht haltbar ist (die Präsentation im Umzug also zur Verbsemantik $\mathrm{zu}$ rechnen ist und sich nicht nur im Einzelfall aus der Referenz ergibt), ${ }^{34}$ gibt es gute Gründe für die Annahme, dass Paulus in 2. Kor 2,14 keine ausführlicher ausgestaltete „fiktionale Erzählung“ von sich und seinen Mitarbeitern $^{35}$ in einer Siegesfeier Gottes in Rom vornimmt.

Bereits das \&́v $\tau \tilde{\omega}$ X werden und bietet wohl eher einen Schlüssel zur Interpretation der Metapher, indem es auf die „in Christus“ stattfindenden Missionsbewegungen der Sachhälfte abzielt. ${ }^{36}$

In der koordinierten Partizipialphrase mehren sich dann die Hinweise, dass Paulus möglicherweise den Aspekt des Geruchs aus dem Umfeld der Rede von einer Prozession übernimmt, dann aber unabhängig von diesem Hintergrund Parallelen zwischen antiken Vorstellungen bezüglich der Eigenschaften von,Geruch“ auf der einen Seite und seiner Evangeliumsverkündigung auf der anderen Seite auslotet. ${ }^{37}$

\subsubsection{Kriegsgefangene in V. 15-16?}

Ebenso problematisch ist die These, dass das Schicksal der Gefangenen auch noch über 2,14 hinaus sogar in 2,15-16 Gegenstand des Diskurses ist. Wer ver-

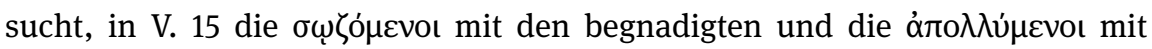
den zur Hinrichtung geführten Gefangenen zu parallelisieren, steht vor dem

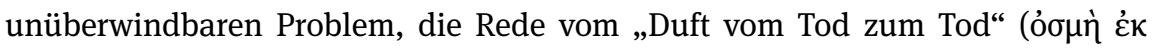

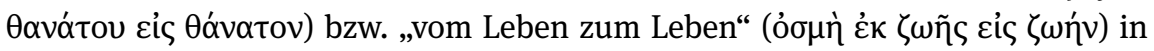

32 Breytenbach, „Proclamation.“

33 Breytenbach, „Proclamation,“ 269. Siehe auch neuerdings Guthrie, „Triumphal Procession“ mit einem noch weiter gehenden Vorschlag. Für ihn ist zwar in der ersten Partizipialkonstruktion vom Umhergeführtwerden die Rede, allerdings sei dabei bereits an die Weihrauchträger gedacht. 34 Siehe hierzu die ausführliche Diskussion der relevanten antiken Vorkommnisse bei Heilig, Paul's Triumph, Kapitel 2, Abschnitt 1 und 2.

35 Zum Plural siehe Heilig, Paul's Triumph, Kapitel 6, Abschnitt 1.

36 Zur Präpositionalphrase siehe Heilig, Paul's Triumph, Kapitel 6, Abschnitt 2.2.

37 Vgl. Heilig, Paul's Triumph, 175-184. 
den Kontext einer solchen Erzählung zu integrieren. Dasselbe gilt für eine ange-

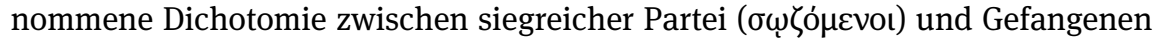
('́)

Während also die Annahme einer die Triumphzugs-Metapher in 2. Kor 2,14 betreffenden narrativen Substruktur den Text in der Tat erhellt, gilt dies nicht, wenn man nach dem Fortwirken der Szene einer Sieges-Prozession im unmittelbaren Fortgang des Textes fragt.

\subsubsection{Ein „neuer Exodus“ in V. 14a?}

Dasselbe muss auch bezüglich solchen Versuchen geschlussfolgert werden, welche eine umgekehrte Betrachtungsweise an den Tag legen und 2. Kor 2,14 besonders die Triumphzugsmetaphorik in V. 14a - von späteren Hinweisen im Text aus zu verstehen versuchen: So argumentiert Scott, dass Ps 67,18-19 eine Verbindung der Idee von Gottes Kriegswagen ( $\alpha \rho \alpha \alpha)$ und Sinai-Offenbarung belege. ${ }^{39} \mathrm{Da}$ im Verb $\theta \rho ı \mu \beta \varepsilon v ́ \omega$ nun die römische quadriga impliziert sei, greife Paulus über dieses Bindeglied nicht nur auf die auf Ez 1 aufbauende Tradition des merkabah-Mystizismus zurück, sondern stelle zugleich implizit Paulus in einen Kontrast mit Mose. ${ }^{40}$

Recht ähnlich ist der Vorschlag von Webb, dass Paulus hier zwar durchaus das Bild des römischen Triumphzuges bemühe, damit aber eigentlich eine genuin jüdische Vorstellung transportieren wolle. Im Hintergrund stehe die bei den Propheten belegte Adaption der Exodus-Erzählung für die Rückkehr aus dem Exil. ${ }^{41}$ Paulus gebrauche also die Triumphzugs-Metaphorik, „to introduce the section on his new covenant ministry (in contrast to Moses’ old covenant ministry).“42

Nun spielt Mose nicht nur in 2. Kor 3 eine prominente Rolle, ${ }^{43}$ sondern es kann in der Tat auch ein gutes Argument für die These geboten werden, dass bereits in der zurückweisenden Frage in 2. Kor 2,16 „Und wer ist hierzu tüchtig?“

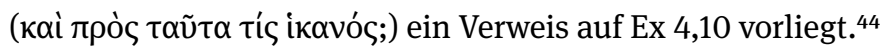

Das Plausibilität der Annahme einer narrativen Substruktur, welche Paulus als zweiten Mose in einem „neuen Exodus“ zum Gegenstand hat und unter der Textoberfläche bis 2. Kor 2,14 zurückreicht, ist dennoch mit einem schwerwie-

38 Siehe hierfür die ausführliche Analyse bei Heilig, Paul's Triumph, 185-190.

39 Scott, „Triumph,“ 268-270.

40 Scott, „Triumph,“ 270.

41 Webb, Returning Home, 72-84.

42 Webb, Returning Home, 82.

43 Siehe die traditionsgeschichtliche Analyse bei Vollenweider, Freiheit, 253-269.

44 Vgl. Heilig, Paul's Triumph, 261. 
genden Problem verbunden: Das Erklärungspotenzial dieser Hypothese ist sehr gering.

Hätte Paulus tatsächlich eine solche Verbindung herstellen wollen, hätten ihm zahlreiche sprachliche Möglichkeiten zur Verfügung gestanden, diesen Zusammenhang anzudeuten oder zumindest offenzulassen (etwa durch Gebrauch

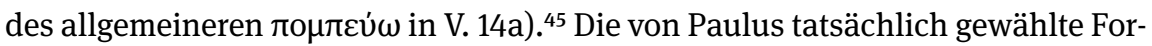
mulierung stellt demgegenüber gerade keine naheliegende Wahl dar, um den von Scott und Webb angenommenen Gedanken auszudrücken. ${ }^{46}$

\subsubsection{Fazit}

Einerseits bekräftigt die Analyse von 2. Kor 2,14 also mit aller Vehemenz die Berechtigung einer narratologischen Perspektive auf die Paulusbriefe. Der Übergang von 2,12-13 zu 2,14 bestätigt, (a) dass Paulus als Erzähler seine Leserschaft nicht aus den Augen verliert, sondern sehr bewusst erzählt und sich über die Wirkung seines Erzählens in der konkreten Kommunikationssituation Gedanken macht.

Das (b) Übergreifen des Narrativs der Reisebewegungen von 2,13 auf 2,14 ist somit kein Zufall, sondern stellt vielmehr eine sorgfältig auf die Adressaten abgestimmte Metapher dar, um deren Reaktion zu steuern. Die Annahme dieser narrativen Substruktur stellt sich gerade hier als heuristisch äußerst wertvoll heraus, da nur sie eine befriedigende Erklärung des Übergangs von 2,13 zu 2,14 bietet. ${ }^{47}$

45 Siehe zum semantisch viel spezifischeren $\theta \rho ı \alpha \mu \varepsilon v \dot{\omega} \omega$ und seiner geringen „Erwartbarkeit“ im Rahmen des Verständnisrahmens von Scott und Webb siehe Heilig, Paul's Triumph, Kapitel 5, Abschnitt 1.2.

46 Heilig, Paul's Triumph, 202-206.

47 Duff, Moses, 38-41 ist durchaus in seiner Kritik bisheriger Erklärungsversuche, die keine Briefteilungshypothese voraussetzen, zuzustimmen: Sie mögen unter Umständen erklären können, weshalb die versöhnliche Schilderung der positiven Nachricht des Titus erst nach 7,4 kommt (so Thrall, Second Epistle, 24), doch lassen sie die Frage offen, weshalb er die Erzählung überhaupt vor 2,14 beginnt und nicht die ganze Erzählung erst nach dieser Passage liefert. Duff ignoriert allerdings den Ansatz von Hafemann, Suffering, 84, welcher die Danksagung als auf einen Einwand zu 2,12-13 reagierend versteht. In diesem Fall trifft Duffs Kritik nicht, da die begonnene Erzählung selbst die Apologie auslöst. Hafemann ist über weite Strecken zuzustimmen, auch wenn sein Fokus auf apostolisches „Leiden“ als Stein des Anstoßes zu allgemein scheint: Es ist nicht klar, weshalb die Sorge um Titus eine unmittelbare Antwort benötigt hätte (vgl. Hafemann, Suffering, 84: „His anxiety over Titus ... makes it necessary to remind the Corinthians immediately of the essential role his suffering plays in his apostolic ministry."), wo doch bereits 1,8-9 gegenüber 2,13 viel schärfer auf diesen Aspekt des Leidens fokussiert. Auch von der Triumphzugs-Metapher selbst her kann der Vorschlag kritisiert werden: Da die Verbsemantik keine Hinrichtung impliziert (Heilig, Paul's Triumph, 100) und die Rede vom Geruch kaum Opfersymbolik darstellt (Heilig, Paul's Triumph, Kapitel 4, Abschnitt 3.2) ist eine Fokussierung auf 
Andererseits zeigt sich an dieser Passage jedoch auch, dass das Postulat narrativer Substrukturen einer sorgfältigen Prüfung des Erklärungspotenzials dieser Hypothese bedarf. Dies gilt für jeden analysierten Textabschnitt und für jede postulierte Substruktur individuell. Einen Freifahrtschein für die Annahme narrativer Substrukturen darf man aus der hier verfolgten Argumentation für den Wert einer narratologischen Perspektive auf die Paulusbriefe daher keinesfalls ableiten.

Auch bedarf es im Hinblick auf die Evaluation der Hypothese narrativer Substrukturen am Einzeltext mehr als nur der Identifizierung einzelner Aspekte im Text, die gut zur vorgebrachten These „passen“ würden. Vielmehr muss - vergleichend zu Alternativhypothesen - abgewogen werden, ob die von Paulus gewählte Formulierung tatsächlich im Rahmen dieser Annahme erwartbar wäre. Für die exegetische Praxis bedeutet dies, dass gerade die Analyse, die nur den vorliegenden Text im Blick hat, diesen unmöglich angemessen erfassen kann. Was für eine Einschätzung erwartbarer kommunikativer Lösungen vielmehr erforderlich ist, ist die Kenntnis der nicht im Text gewählten Ausdrucksmöglichkeiten. Die im Gefolge von Hays narrative Substrukturen - ebenso wie Echos ${ }^{48}$ - postulierenden Arbeiten lassen diesen Aspekt bisher leider nahezu durchgehend vermissen.

den Aspekt des Leidens nicht plausibel (vgl. auch ausführlich die Interaktion mit Hafemann, Suffering bei Heilig, Paul's Triumph, 247-254).

48 Dieser Vorbehalt gilt generell gegenüber Thesen intertextueller Beziehungen. Wie bei Heilig und Heilig, „Historical Methodology,“ 141-142 diskutiert, werden demgegenüber häufig „Echos“ alttestamentlicher Texte aufgrund von lexikalischen Parallelen postuliert, ohne dass eine entsprechende Prüfung der die Wahrscheinlichkeit der Hypothese konstituierenden Parameter durchgeführt würde. 